\title{
Serum Lipid and Fatty Acid Profiles in Adriamycin-Treated Rats after Administration of L-Carnitine
}

\author{
YOUNG MI HONG, HAE SOON KIM, AND HYE-RAN YOON \\ Department of Pediatrics, College of Medicine, Ewha Womans University, Korea [Y.M.H., H.S.K.]; and \\ Metabolic Disease Detection Laboratory, Seoul Medical Science Institute, Korea [H-R.Y.]
}

\begin{abstract}
ABS
Cardiomyopathy induced by Adriamycin (ADR) is a cause of
congestive heart failure. Recently, it has been suggested that
ADR inhibits the carnitine palmitoyltransferase system (CPT I)
and consequently the transport of long-chain fatty acids across
mitochondrial membranes. This study was devised to ascertain
how ADR affects serum lipid and fatty acid metabolism in rats
given ADR with and without L-carnitine supplementation. Male
Sprague-Dawley rats were divided into four groups. The first
group was the control. The second group was given intraperito-
neal injections of ADR (5 mg/kg) twice a week over a period of
2 wk. The third group received the same dose of ADR plus
L-carnitine (200 mg/kg). The fourth group was injected with
L-carnitine only. Serum lipids (total cholesterol, triglyceride,
HDL cholesterol, and LDL cholesterol) and fatty acid levels were
determined on the first, eighth, and 15 th d after injection of ADR.
ADR caused an increas of serum total cholester, triglyceride, ADR caused an increase of serum total cholesterol, triglyceride,
\end{abstract}

and LDL cholesterol compared with the control group. HDL cholesterol was similar between two groups. Similarly, total fatty acids, especially C16-C18 fatty acids, were significantly elevated after injection of ADR. Striking reduction in these substances was observed when L-carnitine was added $(p<0.05)$. This study is the first report regarding the reversal effect of $\mathrm{L}$-carnitine in connection with FFA profiles (C6-C18) in the serum of ADRinduced cardiomyopathic rats. This study also supports the view that ADR causes cardiomyopathy because it interferes with fatty acid metabolism, and we hypothesize that there is a possible protective effect of L-carnitine. (Pediatr Res 51: 249-255, 2002)

Abbreviations
CDR, Adriamycin
CARN, L-carnitine CPT, carnitine palmitoyltransferase
FFA, free fatty acid

ADR (doxorubicin) is an anthracycline antibiotic and has major clinical significance because of its broad-spectrum antitumor activity. Several studies show a cumulative dosedependent and irreversible cardiotoxicity that limits its usefulness as a broad-spectrum anticancer drug $(1,2)$. One major chronic side effect is the development of cardiomyopathy and ultimately congestive heart failure known as ADR cardiomyopathy (3).

The precise mechanism of this pathogenesis has not been completely defined $(4,5)$. A number of different mechanisms have been proposed for the cardiotoxic effect of ADR (6-13), such as its interaction with nucleic acid and/or with nuclear components, disruption of cardiac-specific effects of gene expression, and low antioxidant defense in tissue such as the heart. An abnormal fatty acid metabolism has been suggested by Sole and Liew and others (14-17). Although alteration of

Received January 23, 2001; accepted May 16, 2001.

Correspondence: Young Mi Hong, M.D., Department of Pediatrics, Ewha Womans University 70, Chongro 6- Ka, Chongro-Ku, Seoul 110-126, Korea; e-mail: hongym@chollian.net

Supported by the National Research Fund of the Korean Ministry of Health and Welfare. fatty acid oxidation has been associated with ADR, the lack of understanding of fatty acid metabolism in this type of cardiotoxicity and other cardiomyopathies has led to conflicting results.

Recently, it has been suggested that ADR may exert at least a part of its cardiotoxicity by inhibition of long-chain fatty acid oxidation in the heart (18). Because long-chain fatty acids are the major substrates for energy production in the aerobic adult myocardium (19), their inhibition is usually associated with cardiomyopathy and congestive heart failure due to deficiency in energy supply and accumulation of their toxic intermediates in cardiac tissues (20). However, there have been no studies showing the effect of CARN on fractionated saturated and unsaturated FFA levels (C6:0-C18:0) in the understanding of the mechanisms of inhibition of long-chain fatty acid oxidation in ADR-induced cardiomyopathy.

Therefore, the present study was devised to determine how ADR affects serum lipid and fatty acid metabolism and what would be the mechanism of the reversal and beneficial effect of CARN against ADR-induced cardiomyopathic rats as a function of ADR dose. 


\section{METHODS}

\section{Animals}

Male Sprague-Dawley rats weighing 250-300 g were purchased from Korean Laboratory Animal Center (Chungbuk, Korea). Rats were allowed free access to standard diet essentially free from CARN derivatives and were given water $a d$ libitum. The animal protocol was approved by Seoul National University's Institutional Care and Animal Use Committee. Animals were killed by decapitation.

\section{ADR Administration Protocol}

Rats were divided into four groups: control, ADR (ADRtreated), ADR + CARN (ADR-treated + CARN), and CARN. ADR (doxorubicin hydrochloride, cumulative dose 5, 15, and $20 \mathrm{mg} / \mathrm{kg}$ ) was administered intraperitoneally (Monday and Thursday) in four equal injections (each containing $5 \mathrm{mg} / \mathrm{kg}$ ADR) to animals in ADR and ADR + CARN groups over a period of $2 \mathrm{wk}$. CARN $\left(200 \mathrm{mg} \cdot \mathrm{kg}^{-1} \cdot \mathrm{d}^{-1}\right)$ was also administered intraperitoneally to ADR +CARN and CARN groups every day over a period of $2 \mathrm{wk}$. The total volume of ADR injected was $1.25 \mathrm{~mL} / \mathrm{kg}$ and that of CARN was $1 \mathrm{~mL} / \mathrm{kg}$. All animals were monitored for as long as $2 \mathrm{wk}$ after the last injection for general appearance, behavior, and mortality. After $2 \mathrm{wk}$ of posttreatment with ADR, congestive and depressed heart failure was seen in the rats. At the end of the 2 -wk posttreatment period, animals were killed by decapitation, and blood was collected from the abdominal aorta into nonheparinized tubes on the first, eighth, and 15th d. Serum was immediately separated by centrifugation and was used for the determination of lipid and FFA.

\section{Lipid Analysis}

Serum total cholesterol, triglycerides, and HDL and LDL cholesterol levels were determined enzymatically using kits obtained from HITACHI 7150 (Hitachi, Japan) and were expressed as milligrams per deciliter of serum. Serum total FFA were determined by assay kit NEFA-M reagent purchased from Shinyang Chemical Co. (Seoul, Korea).

\section{Fatty Acid Analysis}

Standards and reagents. Saturated and unsaturated fatty acids (C8:0, C10:1, C10:0, C12:0, C14:1, C14:0, C16:1, $\mathrm{C} 16: 0, \mathrm{C} 18: 2, \mathrm{C} 18: 1$, and $\mathrm{C} 18: 0$ fatty acids), internal standard (C15:0 fatty acid), and N,O-bis(trimethylsilyl)trifluoroacetamide (BSTFA) were purchased from Sigma Chemical Co. (St. Louis, MO, U.S.A.). Deuterium-labeled internal standards (D15-C8:0, D3-C10:0, and D3-C14:0 fatty acids) were purchased from the Cambridge Isotope Laboratory. Hexane, acetonitrile, dichloromethane, methanol, and sodium tetraborate were all of analytical grade and purchased from Merck (Darmstat, Germany) or Sigma Chemical Co. The purity of all standards exceeded $98 \%$.

Preparation of stock and working solutions. Stock solutions of fatty acids and internal standards were prepared by dissolving $5 \mathrm{mg}$ of each compound in $5 \mathrm{~mL}$ of dichloromethane and $10 \mathrm{mg}$ of each compound in $10 \mathrm{~mL}$ of methanol, respectively. A working mixed solution of all standards in methanol was prepared over a concentration ranging from $25 \mu \mathrm{g} / \mathrm{mL}$ (C8:0-C12:0 fatty acids) to $200 \mu \mathrm{g} / \mathrm{mL}$ (C14:0-C18:0 fatty acids).

Sample preparation and derivatization. A $100-\mu \mathrm{L}$ sample of each internal standard solution (D15-C8:0, D3-C10:0, and D3-C14:0, $25 \mu \mathrm{g} / \mathrm{mL} ; \mathrm{C} 15: 0,50 \mu \mathrm{g} / \mathrm{mL}$ ) was added to $0.2 \mathrm{~mL}$ of plasma. The fatty acids were extracted with $2.0 \mathrm{~mL}$ of hexane and further $1.8 \mathrm{~mL}$ of saturated sodium tetraborate solution. Fatty acids in the hexane phase were extracted with $1.5 \mathrm{~mL}$ of dichloromethane and sodium chloride twice after adjustment to $\mathrm{pH} 1.0$. The lower phase of solution was then centrifuged and evaporated under a gentle nitrogen stream. The dry residue was derivatized with $50 \mu \mathrm{L}$ of BSTFA in $25 \mu \mathrm{L}$ of acetonitrile for $30 \mathrm{~min}$ at $80^{\circ} \mathrm{C}$. After cooling, $3 \mu \mathrm{L}$ of sample was injected in a gas chromatograph-mass selective detector (GC-MSD) split mode (20:1).

Instrumentation. A Hewlett-Packard 5973 GC-MSD (Avondale, U.S.A.) used with an HP-5 column (30 m, $0.25 \mathrm{~mm}$ I.D., $0.25-\mu \mathrm{m}$ film thickness). The injector and transfer line temperatures used were 260 and $300^{\circ} \mathrm{C}$, respectively. The oven temperature was programmed from 80 to $290^{\circ} \mathrm{C} \mathrm{(at} 6^{\circ} \mathrm{C} / \mathrm{min}$ ) and held for $2 \mathrm{~min}$. Helium gas was used as a carrier gas (flow rate, $0.8 \mathrm{~mL} / \mathrm{min})$. The temperature of the ion source was $230^{\circ} \mathrm{C}$, and GC-MSD was operated in the electron impact (EI) ionization mode $(70 \mathrm{eV})$.

Calibration. The calibration standards were prepared over a range of $0.6-5 \mu \mathrm{g}$ (C8:0-C12:0 fatty acids) and 1-10 $\mu \mathrm{g}$ (C14:0-C18:0 fatty acids). To standard solutions, $100 \mu \mathrm{L}$ of 25 $\mu \mathrm{g} / \mathrm{mL}$ (D15-C8:0, D3-C10:0, and D3-C14:0 fatty acids) and $50 \mu \mathrm{g} / \mathrm{mL}(\mathrm{C} 15: 0)$ internal standards were added before extraction. The peak area ratio relative to the internal standards was calculated for FFA, and a linear regression analysis was performed. The quantification of the unsaturated FFA was performed on the basis of the calibration curve of the corresponding saturated FFA.

\section{Statistical Analysis}

Data are expressed as the mean $\pm \mathrm{SD}$ of three separate experiments. Statistical significance was determined using the unpaired $t$ test and ANOVA. The $t$ test was used to determine statistical differences between groups $(p<0.05$ was considered significant).

\section{RESULTS}

Effect of ADR on lipid. Figure 1 shows the effect of increasing the concentration of ADR on the serum cholesterol level. ADR caused elevation of the serum cholesterol level in a concentration-dependent manner. The effect appeared to be greatest on the 15th $\mathrm{d}$. On the first d, the cholesterol level was elevated to approximately $29 \%$ compared with the control, whereas it was elevated approximately $50 \%$ on the 15 th $d$ after exposure to a cumulative dose of $20 \mathrm{mg}$ ADR. ADR did not 


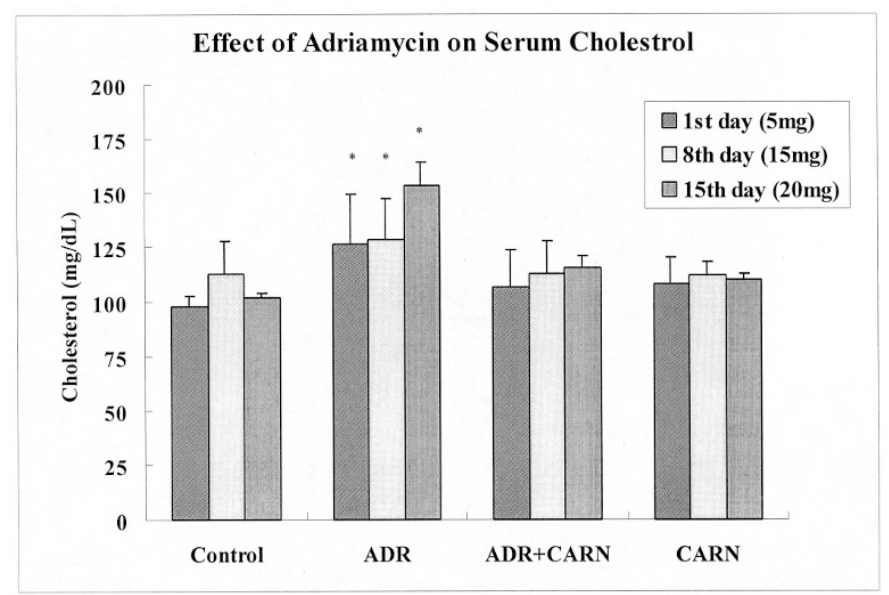

Figure 1. Effect of ADR on serum cholesterol levels (mg/dL) in ADR-treated rat. Values are presented as the mean $\pm \mathrm{SD}$, three determinations. *Indicates a $p$ value $<0.05$ of ADR $v s$ control.

significantly affect the level of serum triglyceride in a dosedependent manner (data not shown).

To assess the effect of ADR on the serum HDL and LDL cholesterol, the concentration of serum HDL and LDL cholesterol was measured. The effect of ADR on LDL cholesterol was greatest on the 15 th $\mathrm{d}$. On the first $\mathrm{d}$, the LDL cholesterol level was elevated approximately $64 \%$, whereas it was elevated approximately $50 \%$ on the 15 th d after exposure to $20 \mathrm{mg}$ ADR. The effect of ADR on serum LDL cholesterol was an elevation of serum LDL cholesterol in a concentrationdependent manner (Fig. 2). The serum HDL cholesterol level was not significantly different among the four groups (data not shown).

Serum total fatty acids significantly increased in the ADR group compared with the control group (Fig. 3). On the first $\mathrm{d}$, the serum total fatty acid level was elevated approximately $220 \%$ after exposure to $5 \mathrm{mg}$ ADR compared with controls and $100 \%$ after exposure to $20 \mathrm{mg}$ ADR by the 15 th d. The effect of ADR on serum total FFA appeared highest on the first $\mathrm{d}$.

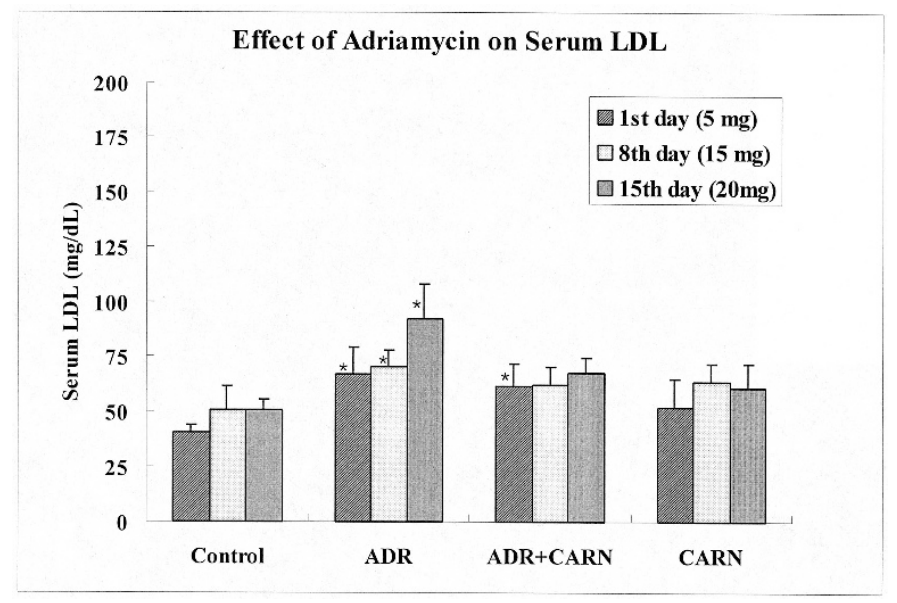

Figure 2. Effect of ADR on serum LDL levels (mg/dL) in ADR-treated rat. Values are presented as the mean $\pm \mathrm{SD}$, three determinations. *Indicates a $p$ value $<0.05$ of ADR vs control.
The addition of CARN to the ADR group almost completely returned then to control levels in serum cholesterol, LDL cholesterol, and total fatty acids except serum triglyceride (Fig. 1, Fig. 2, and Fig. 3).

Effect of ADR on total FFA and FFA profiles. Figures 4, 5, and 6 show the effect of fatty acid metabolism on ADR-treated rats with or without CARN administration. ADR caused significant elevations of long-chain fatty acids such as palmitic (C16:0), linoleic (C18:2), oleic (C18:1), and stearic acids (C18:0) after injection of ADR (Fig. 4-6, Table 1-3). Shortand medium-chain fatty acids such as caproic (C6:0) and caprylic acids (C8:0) did not show significant alterations with ADR (Table 1-3).

CARN dramatically reversed elevated excretion of longchain fatty acids in the ADR group to levels close to the control level, which had been induced by ADR. There was a similar small decrease in the concentration of long-chain fatty acids in the CARN group compared with control (Fig. 4-6, Table 1-3).

\section{DISCUSSION}

The anthracycline glycoside antibiotic ADR is of major importance in cancer chemotherapy. Several studies show a cumulative dose-dependent and irreversible cardiotoxicity that limits its usefulness as a broad-spectrum anticancer drug $(1,2)$. Cardiotoxicity is usually expressed if the total cumulative dose of the drug given is over $550 \mathrm{mg} / \mathrm{m}^{2}$ (21). Cardiotoxicity is the major limiting complication of ADR and affects approximately 30 to $40 \%$ of the patients who receive more than $500 \mathrm{mg} / \mathrm{m}^{2}$ total dose $(3,4)$. Although the precise mechanism of this pathogenesis is not yet completely known $(4,5)$, several hypotheses have been postulated, including the generation of free radicals (21), mitochondrial dysfunction (22), calcium overload (23), inhibition of several membrane-bound molecules $(24,25)$ and altered fatty acid oxidation, the depression of energy metabolism in the cardiac tissue by diverting electrons from the mitochondrial NADH dehydrogenase system (26), the interference with DNA strand separation and helicase activity (6), the initiation of DNA damage via the inhibition of topo-

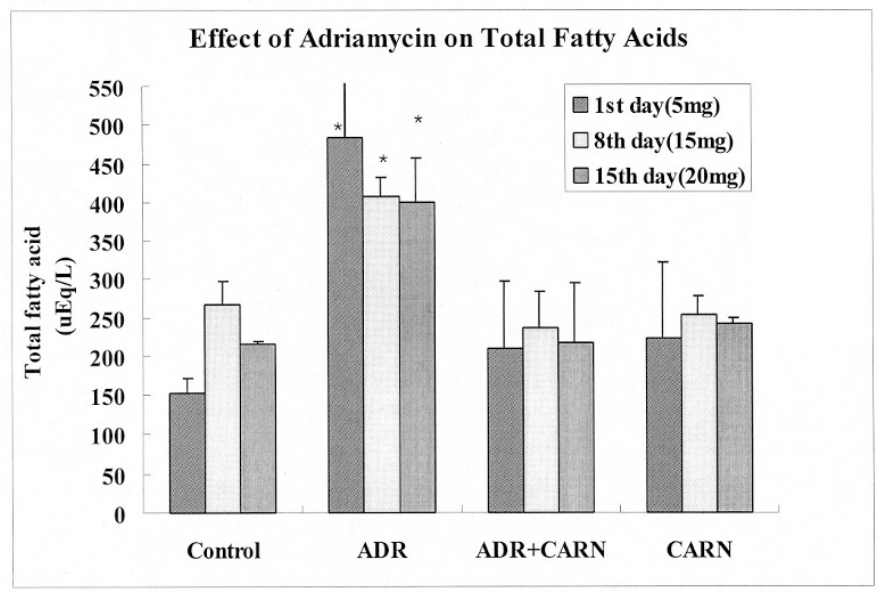

Figure 3. Effect of ADR on serum total fatty acids $(\mu \mathrm{mol} / \mathrm{L})$ in ADR-treated rat. Values are presented as the mean $\pm \mathrm{SD}$, three determinations. *Indicates a $p$ value $<0.05$ of ADR $v$ s control. 


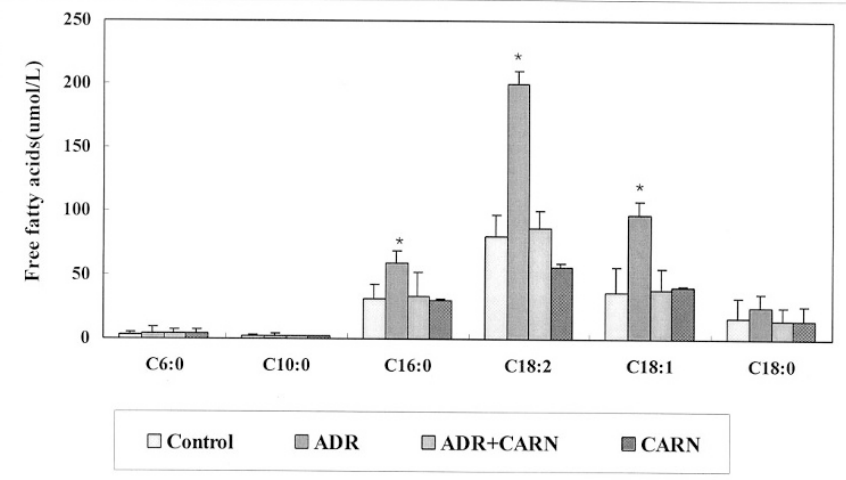

Figure 4. Effect of ADR on serum total fatty acids $(\mu \mathrm{mol} / \mathrm{L})$ in ADR-treated rat on first $\mathrm{d}(5 \mathrm{mg})$. Values are presented as the mean $\pm \mathrm{SD}$, three determinations. *Indicates a $p$ value $<0.05$ of ADR $v s$ control.

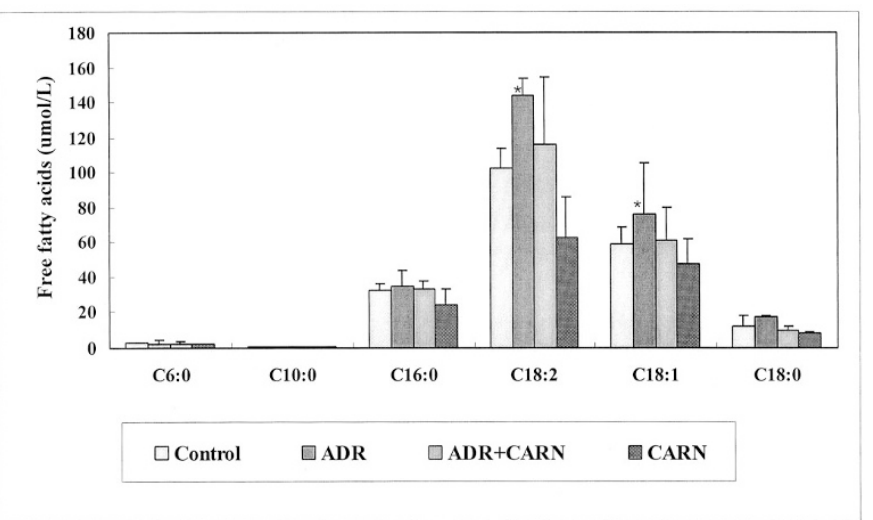

Figure 5. Effect of ADR on serum total fatty acids $(\mu \mathrm{mol} / \mathrm{L})$ in ADR-treated rat on eighth $\mathrm{d}(15 \mathrm{mg})$. Values are presented as the mean $\pm \mathrm{SD}$, three determinations. *Indicates a $p$ value $<0.05$ of ADR vs control.

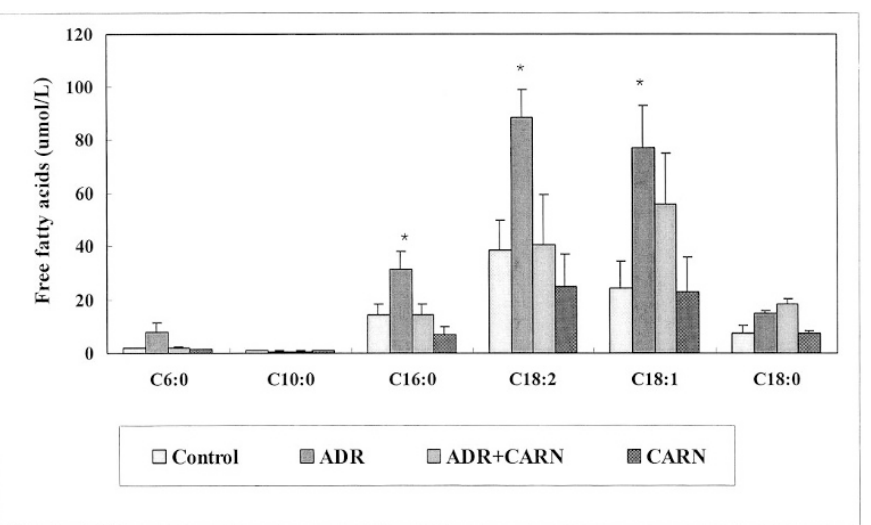

Figure 6. Effect of ADR on serum total fatty acids $(\mu \mathrm{mol} / \mathrm{L})$ in ADR-treated rat on $15 \mathrm{th} \mathrm{d}(20 \mathrm{mg})$. Values are presented as the mean $\pm \mathrm{SD}$, three determinations. *Indicates a $p$ value $<0.05$ of ADR $v s$ control.

isomerase II (6), the reduction in levels of mRNA for the muscle-specific genes cardiac $\alpha$-actin, troponin I, myosin lightchain II, the M isoform of creatine kinase (7), and the decreasein mRNA expression for sarcoplasmic reticulum $\mathrm{Ca}^{2+}$ proteins responsible for calcium handling (8).

Effect of ADR on lipid. Lipid consists of cholesterol (HDL and LDL cholesterol), triglyceride (neutral fat), and phospho- lipids in addition to FFA. Observations of significant elevations of serum cholesterol and LDL cholesterol in our studies showed that ADR reduced the rate of lipolysis in a dosedependent manner, whereas triglyceride and HDL cholesterol were not significantly affected. ADR caused the highest increases of cholesterol and LDL cholesterol, which implies that ADR toxicity is dependent upon the cumulative dose described by Iliskovic et al. (27). We did not find the increased alterations of triglyceride and HDL cholesterol that Iliskovic et al. (27) did. These findings could be the result of the degradation of triglyceride (neutral fat) into FFA, although ADR reduced the rate of lipolysis. On the other hand, if the endogenous triglyceride and lipid pool sizes are increased by ADR, this may dilute the lipid fraction and delay their lipolysis and utilization. Thus, our results show that adverse effects caused by ADR such as hyperlipidemia, which is deleterious for heart function, appear to contribute to ADR-induced heart failure (27).

Effect of ADR on total FFA and FFA profiles. Fatty acids are the preferred energy substrate for the myocardium under normal aerobic conditions (28). The oxidation of fatty acids in mitochondria plays an important role in energy production. During late stages of fasting, fatty acids are used for hepatic ketone synthesis and provide $80 \%$ of total body energy needs via oxidation in muscle. Fatty acids are an important fuel for the heart and serve as an essential source of energy for skeletal muscle during sustained exercise. FFA are released from adipose tissue triglyceride stores and circulate bound to albumin. The oxidation of FFA to $\mathrm{CO}_{2}$ and $\mathrm{H}_{2} \mathrm{O}$ in peripheral tissues, such as muscle, spares glucose consumption and the need to convert body protein to glucose.

Fatty acid oxidation comprises four components: the carnitine cycle, the $\beta$-oxidation cycle, the electron transfer path, and the synthesis of ketone bodies. Long-chain FFA of exogenous and endogenous origin are activated toward their CoA esters in the cytosol. These fatty acyl CoA enter the mitochondria as fatty acylcarnitines via the carnitine cycle. Medium- and shortchain fatty acids enter the mitochondria directly and are activated toward their CoA derivatives in the mitochondrial matrix. Each cycle of the four-step $\beta$-oxidation process shortens the fatty acyl CoA by two carbons until it is completely converted to acetyl CoA. The electron-transfer path transfers some of the energy released in $\beta$-oxidation to the respiratory chain, resulting in the synthesis of ATP. In the liver, most of the acetyl CoA from fatty acid $\beta$-oxidation is used to synthesize the ketone bodies 3 -hydroxybutyrate and acetoacetate. The ketones are then exported for terminal oxidation (chiefly in the brain). In other tissues, such as muscle, the acetyl CoA enters the Krebs' cycle of oxidation and ATP production (Fig. 7).

Changes in fatty acid metabolism may have important influences on myocardial function $(14,17)$ and, thus, have implications for diagnosis and therapy. In this study, the administration of ADR resulted in inhibition of fatty acid oxidation in ADRinduced cardiomyopathic rats, as FFA levels were significantly higher compared with control groups. Especially, total fatty acid alteration was different depending on the fatty acid chain length as a function of the dose of ADR (Fig. 3). 
Table 1. Effect of ADR on serum fatty acids ( $\mu \mathrm{mol} / \mathrm{L})$ in ADR-treated rats on first d (5 $\mathrm{mg})$

\begin{tabular}{crrrr}
\hline FFA & \multicolumn{1}{c}{ Control } & \multicolumn{1}{c}{ ADR } & ADR + CARN & CARN \\
\hline C $8: 0$ & $13.30 \pm 6.90$ & $19.30 \pm 7.70$ & $7.20 \pm 4.20$ & $10.90 \pm 8.50$ \\
C10:1 & $0.10 \pm 0.20$ & $0.30 \pm 0.20$ & $0.30 \pm 0.20$ & $0.20 \pm 0.20$ \\
C12:1 & $0.02 \pm 0.01$ & $0.07 \pm 0.02$ & $0.07 \pm 0.01$ & $0.08 \pm 0.02$ \\
C12:0 & $2.10 \pm 1.50$ & $3.30 \pm 1.10$ & $3.10 \pm 1.10$ & $2.30 \pm 1.70$ \\
C14:2 & $0.30 \pm 0.20$ & $0.50 \pm 0.20$ & $0.40 \pm 0.20$ & $0.40 \pm 0.20$ \\
C14:1 & $0.40 \pm 0.40$ & $0.80 \pm 0.40$ & $9.40 \pm 4.30$ & $0.80 \pm 0.60$ \\
C14:0 & $5.00 \pm 3.40$ & $12.70 \pm 4.60$ & $8.10 \pm 4.80$ & $10.50 \pm 4.90$ \\
C16:1 & $5.00 \pm 4.00$ & $12.60 \pm 11.60$ & $10.50 \pm 4.70$ \\
\hline
\end{tabular}

Values are presented as mean \pm SD of three determinations.

Table 2. Effect of ADR on serum fatty acids ( $\mu \mathrm{mol} / \mathrm{L})$ in ADR-treated rats on eighth d (15 mg)

\begin{tabular}{ccccc}
\hline FFA & Control & ADR & ADR + CARN & CARN \\
\hline C $8: 0$ & $9.00 \pm 2.10$ & $10.40 \pm 6.50$ & $6.70 \pm 0.50$ & $9.90 \pm 1.90$ \\
C10:1 & $0.50 \pm 0.00$ & $0.30 \pm 0.10$ & $0.40 \pm 0.20$ & $0.60 \pm 0.30$ \\
C12:1 & $0.16 \pm 0.02$ & $0.05 \pm 0.01$ & $0.06 \pm 0.02$ & $0.13 \pm 0.06$ \\
C12:0 & $2.60 \pm 1.50$ & $2.70 \pm 0.80$ & $2.10 \pm 0.40$ & $2.50 \pm 2.30$ \\
C14:2 & $0.60 \pm 0.00$ & $0.60 \pm 0.10$ & $0.60 \pm 0.10$ & $0.60 \pm 0.10$ \\
C14:1 & $0.50 \pm 0.40$ & $0.60 \pm 0.10$ & $8.20 \pm 0.10$ & $0.80 \pm 0.60$ \\
C16:0 & $9.90 \pm 3.40$ & $9.20 \pm 2.10$ & $3.50 \pm 0.10$ & $10.60 \pm 4.90$ \\
\hline
\end{tabular}

Values are presented as mean \pm SD of three determinations.

Table 3. Effect of ADR on serum fatty acids ( $\mu \mathrm{mol} / \mathrm{L})$ in ADR-treated rats on 15th d (20 $\mathrm{mg})$

\begin{tabular}{ccccc}
\hline FFA & Control & ADR & ADR + CARN & CARN \\
\hline C $8: 0$ & $9.10 \pm 2.10$ & $6.90 \pm 1.30$ & $1.90 \pm 3.60$ & $8.60 \pm 1.90$ \\
C10:1 & $0.40 \pm 0.00$ & $0.50 \pm 0.30$ & $0.40 \pm 0.20$ & $0.40 \pm 0.30$ \\
C12:1 & $0.10 \pm 0.01$ & $0.05 \pm 0.02$ & $0.13 \pm 0.02$ & $0.13 \pm 0.06$ \\
C12:0 & $2.70 \pm 1.50$ & $2.30 \pm 0.70$ & $1.10 \pm 0.40$ & $2.70 \pm 2.30$ \\
C14:2 & $0.50 \pm 0.00$ & $0.60 \pm 0.10$ & $1.30 \pm 0.10$ & $0.10 \pm 0.10$ \\
C14:1 & $0.80 \pm 0.40$ & $0.70 \pm 0.10$ & $5.70 \pm 0.60$ & $1.60 \pm 0.60$ \\
C14:0 $16: 1$ & $9.90 \pm 3.40$ & $3.20 \pm 1.70$ & $3.20 \pm 0.10$ & $10.20 \pm 2.80$ \\
\hline
\end{tabular}

Values presented as mean \pm SD of three determinations.

In FFA profiles, the excretion of long-chain fatty acids such as palmitic (C16:0), linoleic (C18:2), oleic (C18:1), and stearic acids (C18:0) was highly elevated. This is speculated to be the result of the impairment of long-chain fatty acid oxidation after injection of ADR (Fig. 4-6). The inhibition of oxidation of long-chain fatty acids by ADR is greater than that of mediumand short-chain fatty acids (Fig. 4-6, Table 1-3) in rat sera.

An interesting finding is that the inhibition was more prominent in unsaturated fatty acids than saturated fatty acids. This fits well with the report by Bordoni et al. (29) implicating the disruption of unsaturated fatty-acyl chains in the endoplasmic reticulum, which contains the system catalyzing the desaturation/elongation of fatty acids. Although alteration of fatty acid oxidation is associated with ADR, the exact site of the inhibition remains undetermined. Inhibition of fatty acid metabolism results in the accumulation of toxic intermediates including long-chain fatty acyl-CoA thioesters and long-chain acylcarnitine derivatives (30). Accumulation of these intermediates causes deleterious effects in cardiac tissue and decreased cardiac function similar to that reported in ADR cardiomyopathy.

Two distinct systems of $\beta$-oxidation enzymes are present in mitochondria (31). The first system is responsible for the oxidation of long-chain acyl-CoA esters and is bound to the inner mitochondrial membrane. The second system is respon- sible for the oxidation of short- and medium-chain esters and is located in the mitochondrial matrix. Ironically, our results did not show an elevation of medium- and short-chain fatty acids with ADR treatment. Therefore, we hypothesize that the levels of medium- and short-chain fatty acids are independent of long-chain fatty acid levels unless peroxisomal fatty acid oxidation impairs them. Blocking of long-chain fatty acid oxidation in mitochondria will promote the oxidation of long-chain fatty acid oxidation in peroxisomes. Thus, medium- and shortchain fatty acids could be elevated and cleared by carnitine conjugation because they are able to freely get across the mitochondrial membrane.

Our results do not agree with those of Abdel-aleem et al. (18). Sayed-Ahmed et al. (32) reported that ADR inhibited the oxidation of both octanoate (medium-chain) and butyrate (short-chain) fatty acids in isolated cardiac myocytes. Mediumand short-chain fatty acids such as octanoate and butyrate cross the inner mitochondrial membrane independent of CPT I activity and are also oxidized through the $\beta$-oxidation cycle (18).

Protective effect of CARN. In this study, CARN reduced serum lipid and fatty acid levels in ADR-induced cardiomyopathic rats. The levels of cholesterol and LDL cholesterol in sera were significantly decreased with total fatty acids after the addition of CARN. FFA profiles showed a decrease regardless 
(1) CARNITINE CYCLE

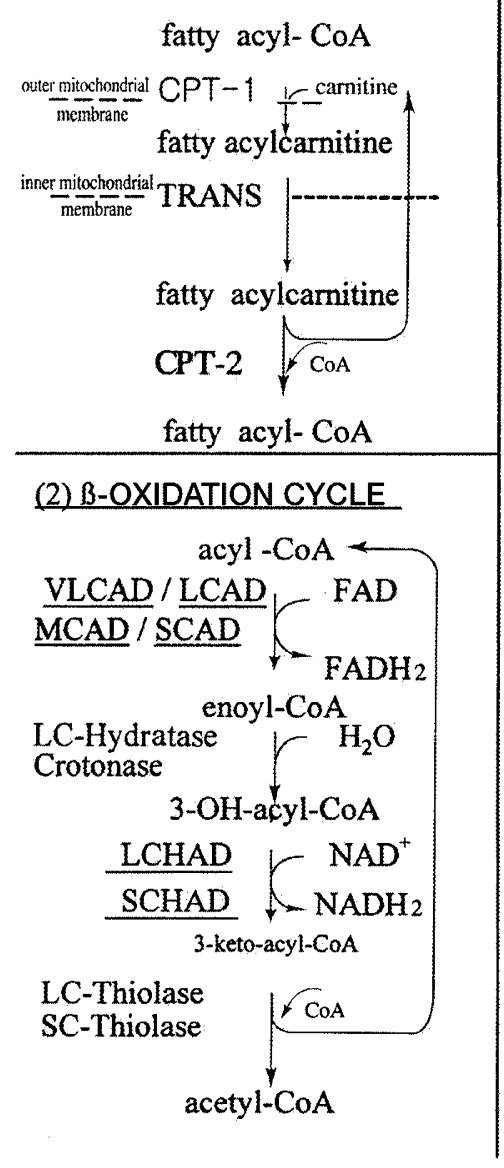

FATTY ACID OXIDATION PATHWAY

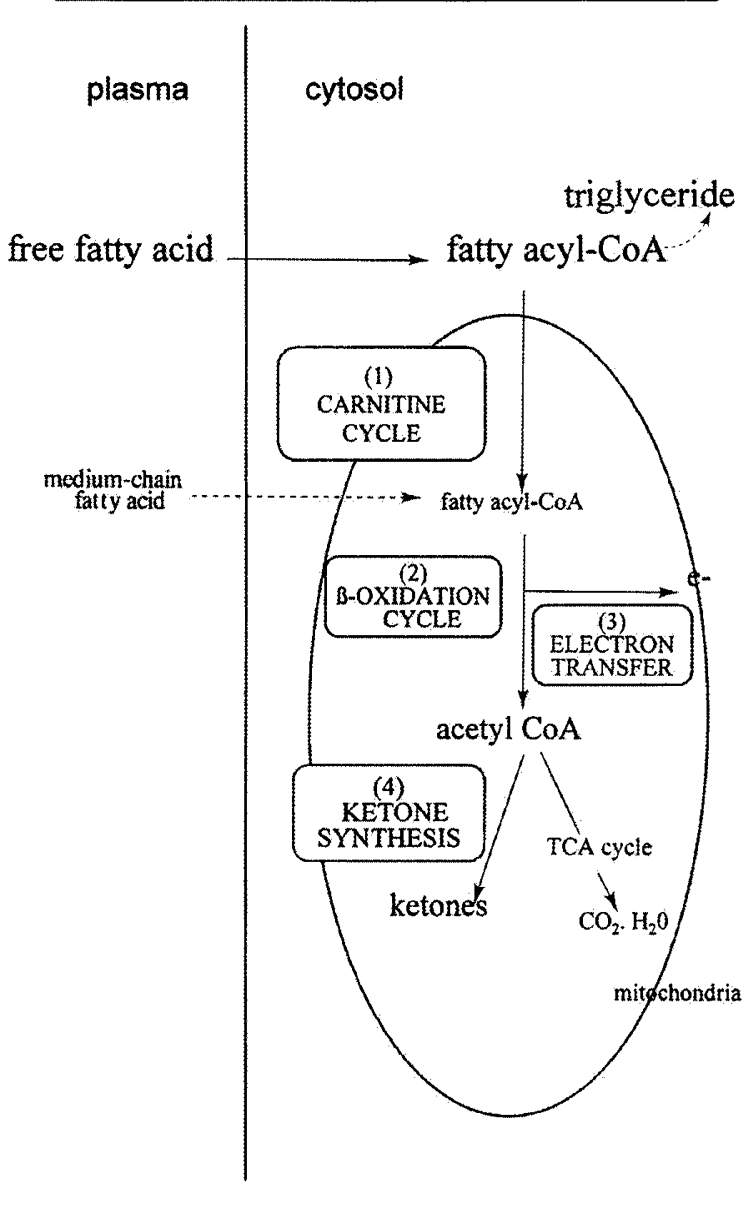

(3) ELECTRON TRANSFER
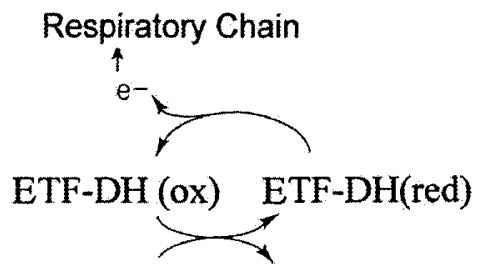

ETF (red) ETF (ox)

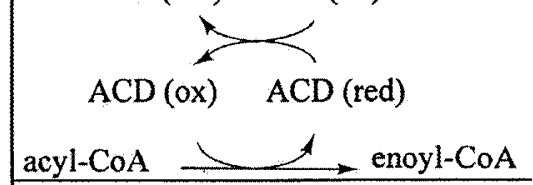

(4) KETONE SYNTHESIS

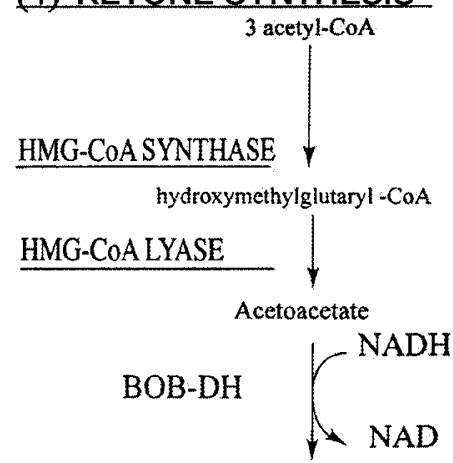

B-hydroxybutyrate

Figure 7. Mitochondrial fatty acid oxidation pathway. In the center panel, the pathway is subdivided into its four major components, which are shown in detail in the side panels. Sites of identified defects are underscored. ETF indicates electron-transfer flavoprotein; ETF- $D H$, ETF dehydrogenase; $F A D$, flavin adenine dinucleotide; $F A D H$, reduced FAD; $H M G$, 3-hydroxy-3-methylglutaryl; $L C A D$, long-chain acyl-CoA dehydrogenase; $M C A D$, medium-chain acyl-CoA dehydrogenase; SCAD, short-chain acyl-CoA dehydrogenase; SCHAD, short-chain 3-hydroxyacyl-CoA dehydrogenase; TCA, tricarboxylic acid; TRANS, carnitine/acylcarnitine translocase; VLCAD, very-long-chain acyl-CoA dehydrogenase. (Adapted from J. Fernandes, J. M. Saudubray, G. van den Berghe (eds) 2000 Inborn Metabolic Disease, 3rd Ed, Springer-Verlag, Berlin, p 140.)

of their chain length after the addition of CARN in the ADRinduced cardiomyopathic rats. This study proved the protective effect of CARN against ADR-induced cardiomyopathy.

This study suggests the protective effect as follows: CARN partially protects the heart against ADR-related cardiotoxicity in a dose-dependent manner as being an essential cofactor for mitochondrial transport and oxidation of long-chain fatty acids (33) as reported by McFalls et al. (34).

It could be related to the CPT system, which is the essential first step in fatty acid oxidation disorders. The possible mechanism by which ADR may inhibit CPT I is by depletion of its cofactor, CARN. Our data demonstrating that CARN administration reverses the inhibition of long-chain fatty acids such as palmitic acid, linoleic acid, and oleic acid oxidation support this hypothesis. Reversal of ADR-induced inhibition of longchain oxidation by CARN suggests that this compound may play a role in enhancing fatty acid metabolism in addition to its role as a cofactor for CPT I.

Another possible mechanism is suggested by previous studies, which demonstrated that ADR interacts with cardiolipin, a specific structural phospholipid of the inner mitochondrial membrane in the heart. CARN may reduce this ADRcardiolipin interaction, preserve the integrity of inner mitochondrial membranes, and, thereby, enhance fatty acid oxidation. CARN could reverse the effects of ADR through CPT I-mediated or CPT I- independent mechanisms.

Considering all the possibilities of the mechanism of the protective effect of carnitine, CARN clearly reversed the effects of ADR. We speculate that the effect of CARN could be through a CPT I-mediated or CPT I-independent mechanism. Therefore, future studies for CPT I and CPT II enzyme analysis in rat hearts are necessary to evaluate the beneficial effect of CARN on ADR-induced cardiomyopathy.

\section{CONCLUSION}

This study is the first report on the reversal effect of CARN in connection with FFA profiles (C6-C18) in the serum of ADR-induced cardiomyopathic rats. This study supports the view that ADR causes cardiomyopathy because of interference 
with fatty acid metabolism, and we hypothesize a possible protective effect of CARN.

\section{REFERENCES}

1. Kantrowitz NE, Bristow MR 1984 Cardiotoxicity of antitumor agents. Prog Cardiovasc Dis 27:195-200

2. Buzadar AV, Marcus C, Smith TL, Blumenschein GR 1985 Early and delayed clinical cardiotoxicity of doxorubicin. Cancer 55:2761-2765

3. Lefrak EA, Pitha J, Rosenheim S, Gottieb JA 1973 A clinicopathologic analysis of adriamycin cardiotoxicity. Cancer 32:302-314

4. Singal PK, Siveski-Iliskovic N, Hill M, Thomas PT, Li T 1995 Combination therapy with probucol prevents adriamycin-induced cardiomyopathy. J Mol Cell Cardiol 27:1055-1063

5. Link G, Tirosh R, Pinson A, Hershko C 1996 Role of iron in the protection of anthracycline cardiotoxicity: identification of heart cell mitochondria as a major site of iron anthracycline interaction. J Lab Clin Med 127:272-278

6. Gewirtz DA 1999 A critical evaluation of the mechanisms of action proposed for the antitumor effects of the anthracycline antibiotics adriamycin and daunorubicin. Biochem Pharmacol 57:727-741

7. Torti SV, Akimoto H, Lin K, Billingham ME, Torti FM 1998 Selective inhibition of muscle gene expression by oxidative stress in cardiac cells. J Mol Cell Cardiol 30:1173-1180

8. Arai M, Tomaru K, Takizaua T, Sekiguchi K, Yokoyama T, Suzuki T, Nagai R 1998 Sarcoplasmic reticulum genes are selectively down-regulated in cardiomyopathy produced by doxorubicin in rabbits. J Mol Cell Cardiol 30:243-254

9. Benjamin RS, Riggs CE, Bauer NR 1993 Pharmacokinetics and metabolism of adriamycin in man. Clin Pharmacol Ther 14:592-600

10. Unverferth DV, Leier CV, Balcerzak SP, Hamlin RL 1985 Usefulness of a free radical scavenger in preventing doxorubicin-induced heart failure in dogs. Am J Cardiol 56:157-161

11. Myers CE, McGuire WP, Liss RH, Ifrim I, Grotzinger K, Young RC 1977 Adriamycin: the role of lipid peroxidation in cardiac toxicity and tumor response. Science 19:165-167

12. Siveski-Iliskovic N, Kaul N, Singal PK 1994 Probucol promotes endogenous antioxidants and provides protection against adriamycin-induced cardiomyopathy in rats. Circulation 89:2829-2835

13. Doroshow JH 1983 Effects of anthracycline antibiotics on oxygen radical formation in rat heart. Cancer Res 43:460-472

14. Sole M, Liew CC 1988 Catecholamines, calcium, and cardiomyopathy. Am J Cardiol $62: 20 \mathrm{G}-24 \mathrm{G}$

15. Whitmer J 1987 Carnitine treatment improves cardiac performance and restores high-energy phosphate pools in cardiomyopathic Syrian hamsters. Circ Res 61:396408
16. Regitz V, Shug A, Fleck E 1990 Defective myocardial carnitine metabolism in congestive heart failure secondary to dilated cardiomyopathy and to coronary, hypertensive, and valvular heart disease. Am J Cardiol 65:755-760

17. Whitmer J 1986 Energy metabolism and mechanical function in perfused hearts of Syrian hamsters with dilated and hypertrophic cardiomyopathies. J Mol Cell Cardiol 18:307-317

18. Abdel-aleem S, el-Merzabani MM, Sayed-Ahmed M, Taylor DA, Lowe JE 1997 Acute and chronic effects of adriamycin on fatty acid oxidation in isolated cardiac myocytes. J Mol Cell Cardiol 29:789-797

19. Neely JR, Morgan HE 1974 Relationship between carbohydrate and lipid metabolism and the energy balance of heart muscle. Annu Rev Physiol 36:413-459

20. Corr PB, Gross RS, Sobel BE 1985 Amphipathic metabolites and membrane dysfunction in ischemic myocardium. Circ Res 55:135-154

21. Singal PK, Deally C, Weinberg L 1987 Subcellular effects of adriamycin in the heart: a concise review. J Mol Cell Cardiol 19:543-553

22. Goormaghtigh E, Brasseur R, Ruysschaert JM 1982 Adriamycin inactivates cytochrome oxidase by exclusion of the enzyme from its cardiolipin essential environment. Biochem Biophys Res Commun 104:314-320

23. Singal P, Pierce G 1986 Adriamycin stimulates low-affinity $\mathrm{Ca}^{2+}$ binding and lipid peroxidation but depresses myocardial function. Am J Physiol 250:H419-H425

24. Law J, Fumagalli A, Schein PS, Rahman A 1985 Selective inhibition of cardiac cyclic nucleotide phosphodiesterases by doxorubicin and daunorubicin. Life Sci 36:589599

25. Singal PK, Panagia V 1984 Direct effects of adriamycin on rat heart sarcolemma. Res Commun Chem Pathol Pharmacol 43:67-77

26. Gille L, Nohl H 1997 Analysis of the molecular mechanism of adriamycin-induced cardiotoxicity. Free Radic Biol Med 23:775-782

27. 1liskovic N, Singal PK 1997 Lipid lowering: an important factor in preventing adriamycin- induced heart failure. Am J Pathol 150:727-734

28. Beanlands RS, Shaikh NA, Wen WH, Dawood F, Ugnat AM, McLaughlin PR, Carere R, Liu PP 1994 Alterations in fatty acid metabolism in adriamycin cardiomyopathy. J Mol Cell Cardiol 26:109-119

29. Bordoni A, Baigi PL, Hrelia S 1999 The impairment of essential fatty acid metabolism as a key factor in doxorubicin-induced damage in cultured rat cardiomyocytes. Biochim Biophys Acta 1440:100-106

30. Karz AM, Messineo FC 1981 Lipid-membrane interactions and the pathogenesis of ischemic damage in the myocardium. Circ Res 48:1-16

31. Nada MA, Rhead WJ, Sprecher H, Schulz H, Roe CR 1995 Evidence for intermediate channeling in mitochondrial $\beta$-oxidation. J Biol Chem 270:530-535

32. Sayed-Ahmed MM, Shouman SA, Rezk BM, Khalifa MH, Osman AM, ELMerzabani MM 2000 Propionyl-L-carnitine as potential protective agent against adriamycin-induced impairment of fatty acid $\beta$-oxidation in isolated heart mitochondria. Pharmacol Res 41:143-150

33. Sayed-Ahmed MM, Shaarawy S, Shouman SA, Osman AM 1999 Reversal of doxorubicin-induced cardiac metabolic damage by L-carnitine. Phamacol Res 39:289-294

34. McFalls EO, Paulson DJ, Gilbert EF, Shug AL 1985 Carnitine protection against adriamycin-induced cardiomyopathy in rats. Life Sci 38:497-505 